\title{
Mental health status among younger generation around Chernobyl
}

Tomoko Masunaga', Alexander Kozlovsky², Anatoly Lyzikov² ${ }^{2}$ Noboru Takamura ${ }^{3}$, Shunichi Yamashit ${ }^{4}$

${ }^{1}$ Graduate School of International Health Development, Nagasaki University, Nagasaki, Japan

${ }^{2}$ Gomel State Medical University, Gomel, The Republic of Belarus

3Department of Global Health, Medicine and Welfare, Nagasaki University Graduate School of Biomedical Sciences, Nagasaki, Japan

${ }^{4}$ Fukushima Medical University, Fukushima, Japan

Submitted: 13 October 2011

Accepted: 27 November 2011

Arch Med Sci 2013; 9, 6: 1114-1116

DOI: $10.5114 /$ aoms.2013.39798

Copyright @ 2013 Termedia \& Banach

\begin{abstract}
Introduction: In order to improve our understanding of how to approach the younger generation around Chernobyl, we screened mental health status among young adults born after the accident living in the Gomel region, Belarus.

Material and methods: We enrolled 697 medical students who were born after the accident. Participants were asked to answer self-administered questionnaires including the General Health Questionnaire (GHQ-12).

Results: GHQ-12 scores were $1.80 \pm 2.28$ (mean \pm SD) among all 697 subjects. When logistic regression analysis was performed with confounding factors, "economic situation" and "association of diseases and/or poor health condition with radiation exposure" were significantly associated with poor mental status.

Conclusions: Our findings suggest that anxiety about radiation exposure among highly educated medical students in the Gomel region, although they were born after the accident, affects their poor mental health status.
\end{abstract}

Key words: Chernobyl, Fukushima, Mental health, General Health Questionnaire-12.

\section{Introduction}

Twenty-five years have passed since the Chernobyl Nuclear Power Plant (CNPP) disaster. In addition to the clear evidence epidemiologically demonstrated of rapid increase of childhood thyroid cancers, the Chernobyl Forum Report internationally emphasized that psychiatric effect is concered due to social anxiety and psychological damage [1-3]. It is urgently needed to fairly evaluate the mental health status in the young population residing around CNPP, even though they were born after the accident, since they may also experience anxiety about living in "radio-contaminated" areas. For the younger generation who did not experience the CNPP disaster, a positive and promising life style is really desirable and expected.

In this study, we screened the mental health status among young adults born after the accident living in the Gomel region, Belarus, to improve our understanding of how to approach the younger generation around Chernobyl.

\author{
Corresponding author: \\ Noboru Takamura MD, PhD \\ Department of Global Health, \\ Medicine and Welfare \\ Nagasaki University Graduate \\ School of Biomedical Sciences \\ 1-12-4 Sakamoto \\ Nagasaki 852-8523, Japan \\ E-mail: takamura@nagasaki- \\ u.ac.jp
}




\section{Material and methods}

We obtained written, informed consent from 697 medical students who were born after the accident (16-24 years old; 138 men and 559 women) at Gomel State Medical University (Gomel, Belarus) to participate in the study. Among them, 307 (44.0\%) were born in Gomel city, 170 (24.4\%) were born in other areas of the Gomel region, 188 (27.0\%) were born in other regions in Belarus, and 8 (1.1\%) were born in the Russian Federation. Participants were asked to answer self-administered questionnaires including the General Health Questionnaire (GHQ12) and questions regarding demographic, sociological and psychological characteristics. The subjects were divided into two groups by the total GHQ-12 scores, i.e. those with 4 points or above (high GHQ group which reflects poor mental status) and less than 4 (low GHQ group).

\section{Statistical analysis}

Differences of demographic, sociological and psychological variables between the two groups were analyzed by $\chi^{2}$ test. Using those variants which were significantly different between the two groups as independent variables, their effects on GHQ scores (high vs. low) were assessed by logistic regression analysis.

\section{Results}

General Health Questionnaire-12 scores were $1.80 \pm 2.28$ (mean \pm SD) among all 697 subjects. There were significant differences of "economic situation" (difficult vs. not difficult, $p<0.001$ ), "past medical history of cardiovascular diseases" (yes vs. no, $p=0.030$ ) and "association of diseases and/ or poor health condition with radiation exposure" (yes vs. no, $p=0.003$ ) between high and low GHQ groups. When logistic regression analysis was performed with confounding factors, "economic situation" (OR: $0.31, p<0.01$ ) and "association of diseases and/or poor health condition with radiation exposure" (OR: 1.78, $p<0.01$ ) were significantly associated with poor mental status (Table I).

\section{Discussion}

Previously, we observed increase incidence of tuberculosis in the Gomel region after the accident, probably due to the anxiety about radiation safety (radiophobia) and its related health consequences [4]. Our current findings suggest that anxiety about radiation exposure among highly educated medical students in the Gomel region who were born after the accident affects their poor mental health status through not only residing in a truly "radio-contaminated area" but also an imprinted victim consciousness. On the other hand, it is clearly shown that there is no risk of thyroid cancer due to the CNPP accident among children who were born after the accident [5]. To avoid any misunderstanding and prejudice of radiation health effects together with an effort of improvement of economic conditions, continuous challenges at radiation health risk communication are needed even 25 years after the CNPP disaster. Despite the limitation of the survey conducted in the Gomel region, it is clearly demonstrated that currently the younger generation are somehow suffering from poor mental health conditions. Also mental health training of physicians and nurses should be definitely undertaken, in order to improve the mental health status in populations around Chernobyl.

Furthermore, our findings suggest the importance of radiation health risk communication around Fukushima Daiichi nuclear power plant, Japan. After the accident, the Japanese government prohibited the consumption of contaminated foods such as cow milk and vegetables, in order to minimize the internal radiation exposure, according to the experiences of the CNPP disaster [6]. Nevertheless, the accident of the power plant caused "social panic", due to insufficient information on "radiation and health", even outside Fukushima Prefecture [7]. Our current results showed that after the accident, radiation health risk communication is definitely needed in every generation, in order to avoid misunderstanding about radiation exposure and health effects. Since the initial phase of the accident, the authors (N.T. and S.Y.) have been ap-

Table I. Odds ratio (OR) and 95\% confidence interval (CI) for GHQ (high vs. low), as assessed by logistic regression analysis

\begin{tabular}{|lcccc|}
\hline Variable & Unit & OR & $95 \% \mathrm{Cl}$ & Value of $p$ \\
\hline Sex & Men/women & 1.18 & $0.69-2.01$ & 0.54 \\
\hline Digestive diseases & Yes/no & 1.29 & $0.86-1.94$ & 0.22 \\
\hline Circulatory diseases & Yes/no & 1.50 & $0.96-2.38$ & $0.18-0.56$ \\
\hline Economic situation & Not poor/poor & 0.31 & $1.18-2.80$ & $<0.01$ \\
\hline $\begin{array}{l}\text { Association of diseases and/or } \\
\text { poor health condition with radiation exposure }\end{array}$ & Yes/no & 1.81 & & $<.01$ \\
\hline
\end{tabular}


pointed as "Advisors on Radiation Health Risk Management" of Fukushima Prefecture and have implemented radiation health risk communications with the general population in Fukushima Prefecture. Appropriate and continuous communications are definitely needed in Fukushima, in order to avoid misunderstanding and prejudice about radiation health effects, based on the experiences of Chernobyl.

\section{References}

1. World Health Organization. Health effects of the Chernobyl accident and special health care programmes. In: Report of the UN Chernobyl Forum expert group "Health". Bennet B, Repacholi M, Zhanat C (eds.). World Health Organization, Geneva 2006.

2. Mental-health effects of the Chernobyl disaster live on. Lancet 2005; 366: 958.

3. Bromet EJ, Havenaar JM. Psychological and perceived health effects of the Chernobyl disaster: a 20-year review. Health Phys 2007; 93: 516-21.

4. Takamura N, Kryshenko N, Masyakin V, Takashiro H, Yamashta S. Chernobyl-induced radiophobia and the incidence of tuberculosis. Lancet 2000; 356: 257.

5. Shibata Y, Yamashita S, Masyakin VB, Panasyuk GD, Nagataki S. 15 years after Chernobyl: new evidence of thyroid cancer. Lancet 2001; 358: 1965-6.

6. Saenko V, Ivanov V, Tsyb A, et al. The Chernobyl accident and its consequences. Clin Oncol 2011; 23: 234-43.

7. Normie D. Japan disaster. Citizens find radiation far from Fukushima. Science 2011; 332: 6036. 